\title{
Chemical peels in the treatment of acne: patient selection and perspectives
}

This article was published in the following Dove Press journal: Clinical, Cosmetic and Investigational Dermatology

\section{David E Castillo' \\ Jonette E Keri ${ }^{1,2}$}

'Department of Dermatology and Cutaneous Surgery, University of Miami Miller School of Medicine, Miami, FL, USA; ${ }^{2}$ Dermatology Service, Miami VA Hospital, Miami, FL, USA

Video abstract

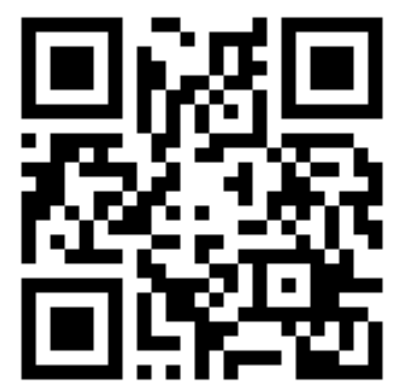

Point your SmartPhone at the code above. If you have a $Q R$ code reader the video abstract will appear. Or use: http://youtu.be/7XlalpPkajk
Correspondence: Jonette E Keri Department of Dermatology and Cutaneous Surgery, University of Miami Miller School of Medicine, 1600 NW 10th Avenue, Miami, FL 33136, USA

Tel +l 3055753167

Fax +l 3052433503

Email JKeri@med.miami.edu

\begin{abstract}
Acne vulgaris is the most common skin disorder in adolescents and young adults. It carries a significant psychological and economic burden to patients and society. A wide range of therapeutic options are available, including topicals and systemic therapies. Chemical peeling is a skin resurfacing procedure intended to regenerate normal skin from the application of exfoliative agents. It has been used for the treatment of acne vulgaris and other skin disorders for decades. There are several chemical agents with variable mechanisms of action, usually classified as superficial, medium, and deep peels. When selecting the patient and the appropriate peel, the dermatologist individualizes therapy, and performs an extensive interview, including past medical history and physical exam. Several host factors can affect the outcome of this procedure, including current psychological state, medications, history of surgery, and immune system, among others. The physician must also be confident that the peel is safe and effective for the target patient. The Fitzpatrick skin type scale is a useful tool to classify patients based on skin color and ability to tan, but also can be used to evaluate preoperative risk of postpeel response and complications. Dark-skinned patients (Fitzpatrick skin type IV-VI), including blacks, Asian, and Hispanic/Latino, are at higher risk of postinflammatory/postpeel hyperpigmentation. When treating these populations, deep chemical peels should be avoided, and preoperative preparation emphasized. There are many studies available in the literature supporting the use of superficial to medium depth peels as adjuvant therapy for acne vulgaris. This review article aims to present the most important factors when selecting a patient for a chemical peel, the evidence behind its safety and efficacy, and special considerations when choosing a specific agent.
\end{abstract}

Keywords: chemical peels, acne, patient perspective, specific population, peel complications

\section{Introduction}

Acne vulgaris is the most common skin disease among adolescents and young adults in the USA. ${ }^{1,2}$ The estimated life-time prevalence is $\sim 80 \%$. ${ }^{3,4}$ The pathogenesis of acne involves several factors, including increased sebum excretion, increased keratinocyte proliferation in the follicular infundibulum, bacterial (Propionibacterium acnes) colonization, and inflammation, and sebaceous glands which are hyper-sensitive to androgens. ${ }^{3,5}$ This disorder is generally considered mild but represents a high economical and psychological burden for the society. Patients experience high levels of anxiety, depression, and low self-esteem which leads to impaired quality of life. ${ }^{6}$ Therefore, treatment should focus on early intervention to decrease the physical and esthetic burden of the disease, and improvement of quality of life. The treatment of 
acne is based on a combination of topical therapies, systemic treatments, chemical peels, dermabrasion, and laser.

Chemical peeling is among the most common cosmetic procedures in medical practice and has been used for decades. It is defined as the application of chemical agents, of variable strength, on the skin that results in controlled destruction of the epidermis and dermis. ${ }^{7}$ The induced exfoliation is followed by dermal and epidermal regeneration from adjacent epithelium and skin adnexa, which results in improved surface texture and appearance of the skin. This is a simple and cost-effective procedure with several dermatological applications. ${ }^{3,7-10}$

Chemicals peels are classified based on the depth of penetration, into superficial (epidermis-papillary dermis), medium (papillary to upper reticular dermis), and deep peels (mid-reticular dermis). ${ }^{7}$ Superficial peels are most commonly used for mild skin disorders such as dyschromia, acne, postinflammatory hyperpigmentation, melasma, and actinic keratosis. ${ }^{8,11}$ Medium depth peels are used for solar keratoses or lentigines, pigmentary disorders, and superficial scars. Deep chemical peels are used for the treatment for photoaging, deep scars or wrinkles, and precancerous skin lesions.

Superficial and medium depth chemical peels are increasingly used by dermatologists in the management of acne vulgaris. Their chemical properties target its different pathophysiological factors. The use of these low cost and safe procedures seem to be supported by evidence,,$^{3,8,9,12}$ but dermatologists should not forget to individualize patients/ treatment to obtain the best outcomes possible.

\section{Patient selection}

When choosing the appropriate peeling agent, physicians must individualize treatment and conduct a complete medical history and skin examination to prevent suboptimal results or complications. The past medical history can reveal factors that could contraindicate the peeling procedure or alter the wound healing process. A list of important factors that should be addressed are is shown in Table 1. There is the notion that patients with acne taking oral isotretinoin should avoid medium to deep peeling agents for at least 6-12 months, to prevent the development of atypical scars or other complications. $^{7,10}$ Isotretinoin is known to produce atrophy of the pilosebaceous unit and to delay the wound healing process. However, a recent systematic review conducted by Spring et al, looking for evidence-based recommendations regarding the safety of cutaneous procedures during or following isotretinoin therapy, found insufficient evidence to delay chemical peels and other skin surgeries. ${ }^{13}$ The authors con- cluded that the patient and physician must weigh risks and benefits of cutaneous procedures in the setting of systemic isotretinoin.

Other medications such as minocycline and oral contraceptives, frequently used in patients with acne, can cause photosensitivity and predispose to hyperpigmentation. Current infection, either bacterial, fungal or viral are contraindications. ${ }^{7,10}$ Herpes simplex virus (HSV) can be reactivated after peeling and delay wound healing if prophylaxis is not done prior to intervention. ${ }^{7}$ Also, immunocompromised patients should be carefully evaluated, as the benefits of skin resurfacing do not outweigh the risk of infection and altered wound healing with scarring seen in these patients. ${ }^{10}$ It is important to emphasize that there is a lack of evidence on the safety of chemical peels during pregnancy and thereby, any peel procedure should be used with caution. ${ }^{14,15}$

The physical exam will determine skin type, degree of photodamage, and presence of other skin disorders. ${ }^{10}$ The Fitzpatrick scale is a useful tool to classify skin based on pigmentation and ability to tan (Table 2). ${ }^{16}$ It can also be used to stratify risk of hyperpigmentation following a peel. ${ }^{10}$ Patients with darker skin types (IV-VI) are at greater risk of developing postpeel/postinflammatory hyperpigmentation, whereas those with type I and II skin do not usually develop this complication.

Finally, physicians should consider the psychological aspects of acne patients. Some patients tend to have a low self-esteem, be anxious about their problem, and might be

Table I Patient selection considerations

Psychologically disturbed patients

Difficult patients, poor doctor-patient relationship

Unrealistic expectations

Current medication: minocycline, nicotine, oral contraceptives (OCP)

Current or previous infection: HSV, bacterial, fungal

Radiation to head or neck

History of hypertrophic or keloid scars

Immunosuppression (HIV)

Recent major surgery (facelift or brow-lift) within 6 months

Pregnancy (avoid)

Note: Information adapted from references. ${ }^{7,10,11}$

Abbreviation: HSV, herpes simplex virus.

Table 2 The Fitzpatrick scale

\begin{tabular}{lll}
\hline Skin type & Skin color & Tanning history \\
\hline I & White & Always burns, never tans \\
II & White & Usually burns, tan with difficulty \\
III & White & Sometimes mild burn, tan average \\
IV & Moderate brown & Rarely burns, tan with ease \\
V & Dark brown & Very rarely burn, tan very easily \\
VI & Black & No burn, tan very easy \\
\hline
\end{tabular}

Note: Reproduced from Roberts WE. Skin type classification systems old and new. Dermatol Clin. 2009;27(4):529-533. With permission from John Wiley and Sons. ${ }^{16}$ 
unrealistic regarding treatment outcomes. A mindful talk with a detailed explanation about the procedure, its implications and possible outcomes will gain the trust of the patient and improve the doctor-patient relationship. This interaction is critical for the success of the procedure. Therefore, dermatologists must be careful when evaluating expectations/concerns and prior experiences with chemical peel and must be realistic about results and complications to achieve patient satisfaction.

\section{Chemical peeling agents}

\section{Salicylic acid (SA)}

$\mathrm{SA}$ is a 2-hydroxybenzoic acid (from willow tree) used for superficial peeling due to its strong keratolytic and comedolytic properties. ${ }^{17}$ It promotes shedding of epidermal cells and due to its lipophilic properties can penetrate comedones and pores to prevent clogging and neutralize bacteria. ${ }^{18}$ It promotes desquamation of the upper lipophilic layers of the stratum corneum. ${ }^{19}$ These chemical properties explain its popularity and success in acne patients..$^{20,21}$ It also has welldocumented anti-inflammatory properties. ${ }^{17}$

Five to $30 \% \mathrm{SA}$ is used for superficial peeling in acne therapy. ${ }^{17}$ At these concentrations, this peel is safe and selflimited; hence, no neutralizing agents are required.

\section{Glycolic acid (GA)}

GA belongs to the group of $\alpha$-hydroxy acids (acids derived from fruit) and is used as a superficial or medium depth peel in acne treatment. ${ }^{20,22} \mathrm{GA}$ is an exfoliative agent that causes epidermolysis with desquamation of the skin by reducing corneocyte adhesion and keratinocyte plugging at the stratum granulosum. ${ }^{9,23}$ Similar to other $\alpha$-hydroxy acids, it leads to a thickened epidermis and dermis with increased collagen and mucopolysaccharide synthesis, and dispersion of melanin. ${ }^{7}$ GA has also been shown to decrease inflammation through bactericidal effects on Propionibacterium acnes, partially explaining its benefits in both inflammatory and noninflammatory acne. ${ }^{24}$

GA peels are commercially available at different concentrations from $20 \%$ to $70 \% .^{22,23}$ The depth of penetration and intensity of GA peels increase with higher concentrations and exposure time. ${ }^{9}$ This agent must be neutralized with an alkaline solution such as sodium bicarbonate or normal saline to stop its exfoliative effects. GA peels have a good safety profile and systemic toxicity is unlikely.

\section{Lactic acid (LA)}

LA is another $\alpha$-hydroxy acid (derived from sour milk or bilberries) used as adjuvant therapy for acne either alone or in combination with other peels. LA decreases the thickness of the stratum corneum by decreasing corneocyte adhesion and removal of dead skin cells. ${ }^{12}$ This leads to the formation of a new stratum corneum. It was shown to decrease melanin synthesis by directly inhibiting the tyrosinase enzyme, thereby explaining its skin whitening effects. ${ }^{25} \mathrm{LA}$ is a safe peel with minor side effects.

\section{Mandelic acid}

Mandelic acid is an aromatic $\alpha$-hydroxy acid widely used for the treatment of mild to moderate acne. ${ }^{18}$ Similar to other $\alpha$-hydroxy acids, mandelic acid induces exfoliation of epidermis by reducing corneocyte adhesion. ${ }^{7,18}$ It is a large molecule that penetrates the skin slowly and possesses antibacterial properties. ${ }^{18,26}$ Therefore, mandelic acid is better tolerated on the skin and is often used as alternative to GA peel. ${ }^{18}$

Mandelic acid peel is formulated in a combination peel with SA (20\% SA, $10 \%$ mandelic acid). ${ }^{3}$

\section{Trichloroacetic acid (TCA)}

TCA is a crystalline inorganic compound that is used as a superficial, medium depth or deep peeling agent depending on the concentration used. ${ }^{12,27}$ TCA causes denaturation of epidermal and dermal proteins, destruction of dermal collagen, and coagulative necrosis of epidermal cells. ${ }^{28,29}$ The clinical effects are the result of dermal structure reorganization and increased collagen, glycosaminoglycans, and elastin in the dermis.

TCA peels (15-50\% concentrations) are available for the treatment of active acne. This peel self-neutralizes and has very low systemic absorption.

\section{Jessner's solution (JS)}

JS is a superficial peeling agent used as adjuvant therapy for acne that is a combination of $14 \%$ SA, 14\% resorcinol, and $14 \%$ LA in $95 \%$ ethanol. ${ }^{2}$ JS peel is commonly used with other peels to increase its penetration. Its mechanisms of action are specific for each ingredient and were described previously but it is generally proposed to break bridges between keratinocytes. Resorcinol is a 1,3-dihydroxybenzene chemically comparable to phenol, which causes disruption of the hydrogen bonds of keratin (keratolysis), disruption of cell membranes (cell death) and is bactericidal. ${ }^{30}$ This combination of compounds makes JS very effective for the treatment of acne.

\section{Kojic acid (KA)}

$\mathrm{KA}$ is a copper chelating agent also known as 5-hydoxyl2-(hydroxymethyl)-4-pyrone, produced by certain fungi. ${ }^{31}$ 
Its skin lightening properties originate from its ability to inhibit the tyrosinase enzyme. It is available at $1 \%-4 \%$ concentrations and is usually used in combination with GA or other lightening agents (arbutin, aloesin, soy extract, etc.) to increase penetration and efficacy. ${ }^{32} \mathrm{KA}$ is frequently found in cosmetic preparations used to treat skin pigmentary disorders. In patients with active acne, KA can be used prior to and following the peel to prevent and treat postinflammatory hyperpigmentation. ${ }^{7}$

\section{Phenol}

Phenol is an aromatic alcohol that has been used as deep chemical peel for years in the dermatology field. It causes epidermolysis, epidermal keratin coagulation, and dermal elastosis. ${ }^{12}$ This leads to activation of dermal fibroblasts and new collagen synthesis. Phenol peels are very effective agents that should be used carefully with appropriate preventive measures due to the high risk of systemic toxicity.

\section{Chemical peels in the treatment of acne vulgaris}

Superficial and medium depth peels have gained popularity in the treatment of acne vulgaris as it is a relatively low cost and safe procedure. They target most of the pathophysiological factors involved in acne vulgaris. Chemical peels break down corneosomes with subsequent exfoliation, induce keratolysis, and have comedolytic effects. ${ }^{12,33}$ They also decrease sebum production and pore size, and have anti-inflammatory and anti-bacterial properties. ${ }^{12}$ Furthermore, chemical peels promote the penetration and absorption of other topical therapies by reducing the barrier effect of the stratum corneum. ${ }^{7,33}$ All these properties and the fact that superficial peels are safe and can be used in combination with other acne medication, make them very popular procedures. The most commonly used superficial and medium depth peeling agents in acne are SA, $\alpha$-hydroxyl acids (GA, LA, etc.), JS, and TCA. ${ }^{12}$

Most studies with respect to using chemical peels for acne have been done on the face. However, in general, the same peeling agents can be used on the back. The chest and neck represent areas where chemical peels may result in scarring, and in general, most people avoid these areas with respect to peels, especially the neck. Acne scarring remains a significant problem with respect to treatment options. Peeling for acne scarring is often done with other interventions such as medical therapy (retinoids, steroids, and silicone dressing), surgical procedures (subcision, skin graft, debulking, cryosurgery, electrodessication, radiation, dermabrasion, microneedling, and fillers), nonablative and ablative laser, light and energy therapy, among others. ${ }^{34} \mathrm{~A}$ detailed review of acne scarring and peeling is outside the scope of this paper.

\section{Specific populations}

\section{Caucasians}

Caucasians have a Fitzpatrick skin type I-III. Their risk of postinflammatory hyperpigmentation or scarring following a peel is low. Hence, most chemical peels can be used safely in these patients. ${ }^{10}$

Lekakh et al demonstrated a significant improvement of active acne lesions in patients with skin type I-II with SA peel alone or in combination with pulsed dye laser. ${ }^{35}$ The authors did not report any minor or serious side effects with the use of SA. Thirty percent SA and GA peels were also evaluated in mild to moderate acne in 20 Caucasian patients by Kessler et al. ${ }^{36}$ Both peels were applied every 2 weeks for a 12-week period. The authors found both peels to be significantly effective, without difference in the reduction of acne lesions. However, they reported that the SA peel had a sustained effect over a longer period. Also, more side effects were reported in the group with the GA peel. Regarding TCA peel, Al Hussein et al also showed that 20\% TCA peel was superior to azelaic acid gel in reducing the number of acne lesions in a comparative study in $2015 .{ }^{37}$ They included 51 patients with mild to moderate acne, and four peeling sessions at 14 days interval were done in an 8-week period. Only mild and transient side effects were reported. Although this study suggests that TCA is an effective and safe peel in mild to moderate acne vulgaris, more studies are required to evaluate its role compared to other agents in this specific population.

\section{Dark-skinned}

Dark-skinned individuals (Fitzpatrick skin type IV-VI) represent a challenging group when selecting appropriate acne therapy. These patients are at greater risk, than light-skinned individuals, of postinflammatory hypo- or hyperpigmentation and scarring. ${ }^{38}$ Therefore, dermatologists must be sure that the peeling agent is safe and effective in dark-skinned patients. ${ }^{39}$ It is generally considered that superficial peels are safe in type III-VI skin (Table 3 ). ${ }^{23}$ Some authors use medium depth peels with good results in skin of color; ${ }^{23}$ however, the practitioner should be experienced in the use of these peels. Deep peels should be avoided due to the risk of hyperpigmentation and scarring.

Back in 1999, Grimes conducted a study evaluating the safety and efficacy of SA in 20 dark-skinned and five Hispanic patients, Fitzpatrick skin type V-VI, with various skin disorders (acne vulgaris, post-inflammatory 
Table 3 Superficial peels safe in dark-skinned patients (Fitzpatrick type IV-VI)

\begin{tabular}{l}
\hline Superficial peel \\
\hline $5 \%-30 \%$ Salicylic acid \\
$30 \%-50 \%$ Glycolic acid \\
Jessner's solution
\end{tabular}

Note: Information adapted from references. . $^{3,60}$

hyperpigmentation, melasma) ${ }^{40}$ The author found that superficial SA was safe and effective in the treatment of acne lesions. Only a few patients presented postpeel hyperpigmentation, which was transient and resolved within 2 weeks. Burns et al demonstrated that serial GA peels effectively reduced postinflammatory hyperpigmentation in Fitzpatrick skin type IV-VI patients. ${ }^{41}$

There are not many studies evaluating chemicals peels for acne treatment in dark-skinned individuals. Most of them have been conducted in Asian patients or have been tested on other cutaneous disorders. However, these two different populations have similar Fitzpatrick skin types and share some of the complications that can derive from it. Both SA and GA peels are safe in these patients but should be used with precaution to avoid complications.

\section{Asians}

Following worldwide trends, acne is one of the most common skin problems among Asians, as shown by many studies. ${ }^{42-44}$ Asians are Fitzpatrick skin type III-V. As mentioned before, their skin type puts them at risk of postpeel complications. The appropriate peeling agent should address both acne lesions and prevent/treat postinflammatory hyperpigmentation. ${ }^{45}$

Several trials have evaluated the safety and efficacy of chemical peels in Asian patients. Most studies focused on SA peel (20\%-30\%) and JS. ${ }^{2,19,46-49}$ Dayal et al reported that both $30 \%$ SA peel alone and JS were effective in the treatment of acne in Asians. ${ }^{2}$ The peels were performed every 2 weeks during a 12-week period for a total of six sessions. SA peel was statistically significantly better than JS for comedonal noninflammatory acne. The decrease in comedonal lesions was noted from baseline and up to 12 weeks of treatment. This can be explained by the strong comedolytic property of SA. Regarding inflammatory lesions, both showed significant improvement without statistically significant difference between them. These results are in concordance with a previous study by Bae et al where both SA peel and JS were found to be very effective and safe for acne in Asian patients. ${ }^{48}$

Variable concentrations of GA peel $(35 \%, 40 \%$, and $50 \%)$ have also been shown to be safe and effective in Asians. ${ }^{50-52}$
Wang et al tested $35 \%$ and $50 \%$ GA peels in 40 Asian patients with moderate to severe acne..$^{50}$ Application of the peel at 3-week intervals for 10 weeks led to significant reduction in comedones, papules, and pustules. The authors noted a decrease in pore size and lighter skin. Only a small number of patients develop side effects such as postinflammatory hyperpigmentation, skin irritation, and HSV reactivation. Seventy percent GA peel was compared to JS in 26 patients with Fitzpatrick skin type III-VI. ${ }^{52}$ Although the group with JS had more side effects, especially more postpeel exfoliation, both led to a significant improvement after three peeling sessions, without significant difference between them. Furthermore, Garg et al found that both 35\% GA peel and 20\% SA/10\% mandelic acid peels (SMPs) were effective in reducing acne lesions and postacne hyperpigmentation in Asians, although the SMPs had slightly better results. ${ }^{26}$ Mild and transient side effects such as burning, stinging sensation, dryness, or photosensitivity were reported. In these studies, GA peel led to a statistically significant reduction in the number of active acne lesions. Importantly, these effects were more pronounced for noninflammatory lesions than for inflammatory acne. ${ }^{48}$

Compared to SA, GA, and JS, it is generally accepted that TCA peel has a greater risk of hyperpigmentation in dark-skinned individuals. Sharquie et al evaluated 35\% TCA peel in 18 Asian patients (Fitzpatrick skin type III-IV) with acne..$^{53}$ One session was performed for every patient with active lesions and three for those with acne scarring. Thirtyfive percent TCA peel significantly reduced papules and pustules after 2 weeks of treatment. There was also a marked reduction in acne scarring. Regarding comedones, only four patients "completely cleared". A few patients experienced postinflammatory hyperpigmentation that cleared up during the 3-month follow-up, besides this, the procedure was well tolerated. ${ }^{53}$ Other studies have also suggested that TCA is a safe peel in dark-skinned individuals. ${ }^{54,55}$ However, it is important to mention that these were conducted on other skin disorders, and most clinicians do not use TCA for the treatment of acne in the darker skin type due to the risk of postpeel hyperpigmentation and scarring.

The level of evidence is enough to support that superficial SA, GA, and JS peels are very safe and effective adjuvant therapies in the management of mild to moderate acne in Asians and dark-skinned individuals. As mentioned before, TCA peel is associated with a greater risk of complications and should be avoided. Regarding other superficial-medium depth peels (LA, phenol, among others), studies have focused on acne scarring or other skin disorders rather than acne vulgaris. More research will determine their role in these populations. 


\section{Hispanic/Latino}

Hispanic/Latinos represent a Fitzpatrick skin type III-VI. Not many studies have evaluated the role of chemical peel as adjuvant therapy for acne in this specific population. However, they share similar skin characteristics to other dark-skinned individuals and the same precautions should be taken when choosing a peeling agent to prevent complications.

\section{Pregnant women}

The safety and tolerability of cosmetic procedures during pregnancy and/or lactation are not well documented. Pregnant women must be counseled about the lack of evidence. ${ }^{15}$ Alpha-hydroxy acids (GA and LA peels) have poor dermal penetration and are generally considered safe. ${ }^{15} \mathrm{SA}$ is category $\mathrm{C}$ and data about safety in pregnancy and lactation are lacking. ${ }^{14}$ However, oral acetylsalicylic acid has been used safely during pregnancy. ${ }^{14}$ If SA peel is under consideration, it should be limited to small areas of the skin (significant dermal penetration) and used with caution..$^{14,56}$ TCA peel has the potential of ocular and oral mucosal absorption and should also be used with caution. ${ }^{15}$ Finally, all medium to deep chemical peels should be avoided due to high absorption and fetal risks. ${ }^{?}$

\section{Peeling complications}

Chemical peel is the most popular noninvasive cosmetic procedure of the last centuries. ${ }^{57}$ If done with the appropriate technique, these are very safe and effective procedures. However, as any other medical intervention, they carry possible complications. A proper understanding of the chemical properties, indications, and procedure for each agent can prevent side effects and unexpected outcomes.

Preoperative patient preparation is essential prior to any chemical peel. A few simple recommendations during this period will yield the best outcome and prevent complications. Patients should be advised to use broad-spectrum sunscreen for at least 2-3 months prior to the peel to decrease the risk of hyperpigmentation or dyschromia. ${ }^{7}$ The use of tretinoin nightly for 6 weeks before the procedure has shown to enhance the process of wound healing and the penetration of the peel. ${ }^{7}$ It should be stopped 48 hours before the peel in light-skinned individuals, whereas dark-skinned patients should stop tretinoin 2-3 weeks prior to decrease the risk of postpeel hyperpigmentation, hypopigmentation, or scarring. ${ }^{7}$ Several other topical agents can be used to minimize complications, including low-dose $\alpha$-hydroxy acid weeks before the peel. The use of bleaching agents (hydroquinone, KA, azelaic acid, aloesin, vitamin $\mathrm{C}$ or $\mathrm{E}$, glabridin, etc.) before and following the peel can treat and decrease dyschromia. ${ }^{7}$ Furthermore, antiviral prophylaxis for every patient undergoing medium to deep peel is highly recommended to prevent HSV reactivation. ${ }^{10}$

Even when proper measures are taken, complications occur. Complications that are common a few days or weeks following the procedure for every type of agent, include swelling, erythema, pain and burning, pruritus, ecchymoses, blistering or infection, milia, and telangiectasia. ${ }^{11,38,57}$ Some patients may develop skin textural changes and appearance of enlarged pores. ${ }^{11}$ These complications are usually mild and resolve within weeks but tend to be more serious for deep peels. A list of serious complications for superficial, medium, and deep chemical peels is provided in Table 4.

Postpeel hyperpigmentation is of special concern. Although any skin type can develop it, dark-skinned patients (skin type IV-VI) are at greater risk. A careful evaluation of the skin type and the appropriate agent will decrease the risk. The point is to avoid deep chemical peels in Fitzpatrick skin type III-VI. ${ }^{20}$ As mentioned before, bleaching agents such as hydroquinone are the standard therapy for postinflammatory hyperpigmentation..$^{38,57}$ The risk of dyschromia and scarring is low for superficial to medium depth peels.

Finally, a few agents have specific risk considerations. SA peel can cause salicylism (tinnitus, nausea/vomiting, rapid breathing or stroke) when used in excess or in combination with other salicylates. ${ }^{38}$ Resorcinol is an agent chemically comparable to phenol. If used in excess, it can cause contact dermatitis and systemic toxicity, leading to nausea/vomiting, diarrhea, tremors, headaches, dizziness, dyspnea, or paralysis. ${ }^{20,57}$ The most feared complications associated with phenol intoxication are listed in Table 4. One of the most common complications of TCA peel is hyperpigmentation, which is more evident in skin types III-VI. ${ }^{57}$ This medium depth peel may also cause accentuation of nevi or lentigines. A few cases of toxic shock syndrome following a chemical peel have also been reported. ${ }^{58,59}$

In conclusion, superficial to medium depth chemical peels are useful adjuvant therapies in acne vulgaris. They effectively target the different factors involved in the development of this common skin disorder and enhance the effects of other topical preparations. The level of evidence supports the use of SA, GA, JS, and TCA peels at variable concentrations. Thereby, this safe and low-cost procedure should be considered in every acne patient. Nonetheless, the benefits and complications of it varies among racial groups, and special attention must be paid when dealing with dark-skinned patients (Fitzpatrick IV-VI). ${ }^{60}$ 
Table 4 Serious complications for superficial, medium, and deep chemical peels

\begin{tabular}{lll}
\hline Superficial peel & Medium depth peel & Deep peel (phenol) \\
\hline Transient hyperpigmentation & Hypo- or hyperpigmentation & Cardiotoxic \\
Other dyschromia & Prolonged erythema & Hepatotoxic \\
Infection & Infections & Nephrotoxic \\
Desquamation & Delayed wound healing & Corneal damage \\
& Scarring & Respiratory depression, laryngeal edema \\
& HSV reactivation & Hypo- or hyperpigmentation \\
& & Scarring \\
& & HSV reactivation
\end{tabular}

Note: Information adapted from references. ${ }^{7,12,20,57}$

Abbreviation: HSV, herpes simplex virus.

\section{Disclosure}

The authors report no conflicts of interest in this work.

\section{References}

1. Bickers DR, Lim HW, Margolis D, et al. The burden of skin diseases: 2004 a joint project of the American Academy of Dermatology Association and the Society for Investigative Dermatology. J Am Acad Dermatol. 2006;55(3):490-500.

2. Dayal S, Amrani A, Sahu P, Jain VK. Jessner's solution vs. 30\% salicylic acid peels: a comparative study of the efficacy and safety in mild-tomoderate acne vulgaris. $J$ Cosmet Dermatol. 2017;16(1):43-51.

3. Al-Talib H, Al-Khateeb A, Hameed A, Murugaiah C. Efficacy and safety of superficial chemical peeling in treatment of active acne vulgaris. $A n$ Bras Dermatol. 2017;92(2):212-216.

4. Gollnick H. Current concepts of the pathogenesis of acne: implications for drug treatment. Drugs. 2003;63(15):1579-1596.

5. Kurokawa I, Oiso N, Kawada A. Adjuvant alternative treatment with chemical peeling and subsequent iontophoresis for postinflammatory hyperpigmentation, erosion with inflamed red papules and non-inflamed atrophic scars in acne vulgaris. $J$ Dermatol. 2017;44(4):401-405.

6. Hosthota A, Bondade S, Basavaraja V. Impact of acne vulgaris on quality of life and self-esteem. Cutis. 2016;98(2):121-124.

7. Singh-Behl D, Tung R. Chemical peels. In: Alam M, Tung R, Gladstone H, editors. Cosmetic Dermatology. Saunders Elsevier; 2009.

8. Rendon MI, Berson DS, Cohen JL, et al. Evidence and considerations in the application of chemical peels in skin disorders and esthetic resurfacing. J Clin Aesthet Dermatol. 2010;3(7):32-43.

9. Clark E, Scerri L. Superficial and medium-depth chemical peels. Clin Dermatol. 2008;26(2):209-218.

10. Tse Y. Choosing the correct peel for the appropriate patient. In: Tung R, Rubin M, editors. Procedures in Cosmetic Dermatology Series: Chemical Peels. 2nd ed. London: Elsevier Health Sciences; 2010:17-21.

11. Costa IM, Damasceno PS, Costa MC, Gomes KG. Review in peeling complications. J Cosmet Dermatol. 2017;16(3):319-326.

12. Kontochristopoulos G, Platsidaki E. Chemical peels in active acne and acne scars. Clin Dermatol. 2017;35(2):179-182.

13. Spring LK, Krakowski AC, Alam M, et al. Isotretinoin and timing of procedural interventions: a systematic review with consensus recommendations. JAMA Dermatol. 2017;153(8):802-809.

14. Lee KC, Korgavkar K, Dufresne RG Jr, Higgins HW 2nd. Safety of cosmetic dermatologic procedures during pregnancy. Dermatol Surg. 2013;39(11):1573-1586.

15. Trivedi MK, Kroumpouzos G, Murase JE. A review of the safety of cosmetic procedures during pregnancy and lactation. Int $J$ Womens Dermatol. 2017;3(1):6-10.

16. Roberts WE. Skin type classification systems old and new. Dermatol Clin. 2009;27(4):529-533.

17. Arif T. Salicylic acid as a peeling agent: a comprehensive review. Clin Cosmet Investig Dermatol. 2015;8:455-461.
18. Dewandre L, Tenenbaum A. The chemistry of peels: a hypothesis of action mechanisms and a proposal of a new classification of chemical peelings. In: Tung R, Rubin M, editors. Procedures in Cosmetic Dermatology Series: Chemical Peels. 2nd ed. London: Elsevier Health Sciences; 2010:1-16.

19. Lee HS, Kim IH. Salicylic acid peels for the treatment of acne vulgaris in Asian patients. Dermatol Surg. 2003;29(12):1196-1199.

20. Handog EB, Datuin MS, Singzon IA. Chemical peels for acne and acne scars in asians: evidence based review. J Cutan Aesthet Surg. 2012;5(4):239-246.

21. Brackett W. The chemistry of salicylic acid. Cosmet Derm. 1997;4:5-6.

22. Sharad J. Glycolic acid peel therapy - a current review. Clin Cosmet Investig Dermatol. 2013;6:281-288.

23. Salam A, Dadzie O, Galadari H. Chemical peeling in ethnic skin: an update. Br J Dermatol. 2013;169(Suppl 3):82-90.

24. Takenaka Y, Hayashi N, Takeda M, Ashikaga S, Kawashima M. Glycolic acid chemical peeling improves inflammatory acne eruptions through its inhibitory and bactericidal effects on Propionibacterium acnes. J Dermatol. 2012;39(4):350-354.

25. Usuki A, Ohashi A, Sato H, et al. The inhibitory effect of glycolic acid and lactic acid on melanin synthesis in melanoma cells. Exp Dermatol. 2003;12(Suppl 2):43-50.

26. Garg VK, Sinha S, Sarkar R. Glycolic acid peels versus salicylicmandelic acid peels in active acne vulgaris and post-acne scarring and hyperpigmentation: a comparative study. Dermatol Surg. 2009;35(1): 59-65.

27. Dinner M, Artz JS. The art of the trichloroacetic acid chemical peel. Clin Plast Surg. 1998;25(1):53-62.

28. Brody HJ. Variations and comparisons in medium-depth chemical peeling. J Dermatol Surg Oncol. 1989;15(9):953-963.

29. Lee JB, Chung WG, Kwahck H, Lee KH. Focal treatment of acne scars with trichloroacetic acid: chemical reconstruction of skin scars method. Dermatol Surg. 2002;28(11):1017-1021.

30. Karam PG. 50\% resorcinol peel. Int J Dermatol. 1993;32(8):569-574.

31. Draelos ZD, Yatskayer M, Bhushan P, Pillai S, Oresajo C. Evaluation of a kojic acid, emblica extract, and glycolic acid formulation compared with hydroquinone $4 \%$ for skin lightening. Cutis. 2010;86(3): $153-158$.

32. Davis EC, CallenderVD. Postinflammatory hyperpigmentation: a review of the epidemiology, clinical features, and treatment options in skin of color. J Clin Aesthet Dermatol. 2010;3(7):20-31.

33. Dréno B, Fischer T, Perosino E, et al. Expert opinion: efficacy of superficial chemical peels in active acne management: what can we learn from the literature today? Evidence-based recommendations. $J$ Eur Acad Dermatol Venereol. 2011;25(6):695-704.

34. Rivera AE. Acne scarring: a review and current treatment modalities. J Am Acad Dermatol. 2008;59(4):659-676.

35. Lekakh O, Mahoney AM, Novice K, et al. Treatment of acne vulgaris with salicylic acid chemical peel and pulsed dye laser: a split face, rater-blinded, randomized controlled trial. J Lasers Med Sci. 2015;6(4):167-170. 
36. Kessler E, Flanagan K, Chia C, Rogers C, Glaser DA. Comparison of alpha- and beta-hydroxy acid chemical peels in the treatment of mild to moderately severe facial acne vulgaris. Dermatol Surg. 2008;34(1):45-50.

37. Al Hussein S, Buicu F, Maier D, et al. Eficacy of trichloroacetic acid peel versus $15 \%$ topical azelaic acid gel in the treatment of acne vulgaris - a comparative study. Acta Medica Marisiensis. 2015;61:25-30.

38. Coleman K, Coleman W 3rd. Complications. In: Tung R, Rubin M, editors. Procedures in Cosmetic Dermatology Series: Chemical Peels. 2nd ed. London: Elsevier Health Sciences; 2010:173-182.

39. Roberts WE. Chemical peeling in ethnic/dark skin. Dermatol Ther. 2004;17(2):196-205.

40. Grimes PE. The safety and efficacy of salicylic acid chemical peels in darker racial-ethnic groups. Dermatol Surg. 1999;25(1):18-22.

41. Burns RL, Prevost-Blank PL, Lawry MA, et al. Glycolic acid peels for postinflammatory hyperpigmentation in black patients. A comparative study. Dermatol Surg. 1997;23(3):171-174.

42. Yeung CK, Teo LH, Xiang LH, Chan HH. A community-based epidemiological study of acne vulgaris in Hong Kong adolescents. Acta Derm Venereol. 2002;82(2):104-107.

43. Shah SK, Bhanusali DG, Sachdev A, Geria AN, Alexis AF. A survey of skin conditions and concerns in South Asian Americans: a communitybased study. J Drugs Dermatol. 2011;10(5):524-528.

44. Chua-Ty G, Goh C, Koh S. Pattern of skin diseases at the National Skin Centre (Singapore) from 1989-1990. Int J Dermatol. 1992;31(8): 555-559.

45. Davis EC, Callender VD. A review of acne in ethnic skin: pathogenesis, clinical manifestations, and management strategies. J Clin Aesthet Dermatol. 2010;3(4):24-38.

46. Hashimoto Y, Suga Y, Mizuno Y, et al. Salicylicacid peels in polyethylene glycol vehicle for the treatment of comedogenic acne in Japanese patients. Dermatol Surg. 2008;34(2):276-279.

47. Dainichi T, Ueda S, Imayama S, Furue M. Excellent clinical results with a new preparation for chemical peeling in acne: $30 \%$ salicylic acid in polyethylene glycol vehicle. Dermatol Surg. 2008;34(7):891-899.

48. Bae BG, Park CO, Shin H, et al. Salicylic acid peels versus Jessner's solution for acne vulgaris: a comparative study. Dermatol Surg. 2013;39(2):248-253.
49. Jaffary F, Faghihi G, Saraeian S, Hosseini SM. Comparison the effectiveness of pyruvic acid $50 \%$ and salicylic acid $30 \%$ in the treatment of acne. J Res Med Sci. 2016;21:31.

50. Wang CM, Huang CL, Hu CT, Chan HL. The effect of glycolic acid on the treatment of acne in Asian skin. Dermatol Surg. 1997;23(1):23-29.

51. Kaminaka C, Uede M, Matsunaka H, Furukawa F, Yamomoto Y. Clinical evaluation of glycolic acid chemical peeling in patients with acne vulgaris: a randomized, double-blind, placebo-controlled, split-face comparative study. Dermatol Surg. 2014;40(3):314-322.

52. Kim SW, Moon SE, Kim JA, Eun HC. Glycolic acid versus Jessner's solution: which is better for facial acne patients? A randomized prospective clinical trial ofsplit-face model therapy. Dermatol Surg. 1999;25(4):270-273.

53. Sharquie K, Noaimi A, Al-Janabi E. Treatment of active acne vulgaris by chemical peeling using tca 35\%. JCDSA. 2013;3:32-35.

54. Puri N. Efficacy of modified Jessner's peel and 20\% TCA versus $20 \%$ TCA peel alone for the treatment of acne scars. J Cutan Aesthet Surg. 2015;8(1):42-45.

55. Sarkar R, Bansal S, Garg VK. Chemical peels for melasma in darkskinned patients. J Cutan Aesthet Surg. 2012;5(4):247-253.

56. Murase JE, Heller MM, Butler DC. Safety of dermatologic medications in pregnancy and lactation: part I. Pregnancy. J Am Acad Dermatol. 2014;70(3):401.e1-401.e14.

57. Nikalji N, Godse K, Sakhiya J, Patil S, Nadkarni N. Complications of medium depth and deep chemical peels. J Cutan Aesthet Surg. 2012;5(4): 254-260.

58. Dmytryshyn JR, Gribble MJ, Kassen BO. Chemical face peel complicated by toxic shock syndrome. A case report. Arch Otolaryngol. 1983;109(3):170-171.

59. LoVerme WE, Drapkin MS, Courtiss EH, Wilson RM. Toxic shock syndrome after chemical face peel. Plast Reconstr Surg. 1987;80(1): 115-118.

60. Hexsel D, Hexsel C, Fernandes J, Rullan P. Peeling in darker skin types. In: Tung R, Rubin M, editors. Procedures in Cosmetic Dermatology Series: Chemical Peels. 2nd ed. London: Elsevier Health Sciences; 2010:89-99.
Clinical, Cosmetic and Investigational Dermatology

\section{Publish your work in this journal}

Clinical, Cosmetic and Investigational Dermatology is an international, peer-reviewed, open access, online journal that focuses on the latest clinical and experimental research in all aspects of skin disease and cosmetic interventions. This journal is included on PubMed. The manuscript management system is completely online

\section{Dovepress}

and includes a very quick and fair peer-review system, which is all easy to use. Visit http://www.dovepress.com/testimonials.php to read real quotes from published authors 